\title{
Hormone Replacement Therapy and Dry Eye in Post Menopausal Women: Study in a Tertiary Centre in Malaysia
}

Norshamsiah M. Din', Siti H. B. Sa'aid², Lam C. Shen², Yap S. Shu², Marina B. A. Rahman², Aini B. M. Azmi², Chang S. Thing², Mushawiahti Mustapha', Ropilah A. Rahman'.

\begin{abstract}
.
Background: Menopause involves decrease in the body estrogen level. There are many disorders associated with estrogen deficiency state. Postmenopausal women frequently report dry eye symptoms due to the decrease in the level of circulating estrogen hormone. Hormone replacement therapy (HRT) is given to alleviate some of the menopausal effects. This study aimed to compare the tear production between postmenopausal women treated with or without HRT. Methods: This cross-sectional study was performed on 140 participants attending various clinics in Universiti Kebangsaan Malaysia Medical Centre (UKMMC) namely the obstetric and gynecology, Surgery, Orthopedic and Otorhinolaryngology clinics over a period of 3 months. The subjects were either with or without HRT. Schirmer's Test strip was used to measure the tear quantity. Dry eye was defined when the length of the moistened area on the strip is less than $5 \mathrm{~mm}$. Results: The results showed significant difference in tear quantity in postmenopausal women treated with or without HRT ( $p=0.003)$. No correlation was observed between tear quantity and duration of HRT consumption, $(p=0.217)$.No significant correlation was also found between the tear quantity and duration of menopause $(\mathrm{p}=0.150)$. Conclusions: Our results suggested that HRT helps in improving tear production in postmenopausal women regardless of the duration of HRT consumption or menopause However, duration of HRT consumption or menopause exert no significant effect on the tear quantity.
\end{abstract}

Keywords: Postmenopausal; Women; Hormone Replacement Therapy; Dry Eye Syndrome (Source: MeSH, NLM).

\section{Introduction.}

Dry eye syndrome is one of the most common clinical entities encountered by eye care professionals, especially among middle-aged and older adults. Dry eye syndrome, also known as keratoconjunctivitissicca, or more recently dysfunctional tear syndrome, has been defined in various ways as the understanding of the disease process evolved. Dry eye syndrome is due to disturbances of the lacrimal functional unit, which comprises of lacrimal glands, ocular surface (cornea and conjunctiva), eyelids, meibomian glands, and associated sensory and motor nervescausing alterations in the volume, composition, distribution or clearance of the tear film.

Menopause is defined as a phase during which there is complete menstrual cessation for at least one year with elevated Follicular Stimulating Hormone (FSH) serum levels $(>40 \mathrm{IU} / \mathrm{L}){ }^{2}$ The hormonal changes may lead to decreased tear production which is the root of dry eye syndro- me. About $60 \%$ of menopausal women are affected by dry eyes. ${ }^{3}$ The symptoms of dry eye, namely dryness, itchiness, photophobia, blurring of vision, foreign body sensation and tearing may cause considerable discomfort to patients and subsequently interfere with their quality of lifeUntreated dry eye syndrome increase the susceptibility of the eyes towards infection as well as causing serious visual impairment.' HRT has been well accepted to improve the quality of life among the postmenopausal women. ${ }^{4}$

Dry eye syndrome is diagnosed by multiple methods such as non-invasive tear break up time, Schirmer's test and fluorescein clearance test. ${ }^{5}$ Schirmer's Test (๔ Haag-Streit UK) remains the most popular and practical method in many clinical settings as it was also reported to correlate well with the basal tear turnover and tear flow. ${ }^{6}$ It is also convenient and cost-effective. At a cut off value of $5 \mathrm{~mm}$, the sensitivity and specificity were reported to be $80 \%$ and $53 \%$, respectively. ${ }^{7}$ 
This study aimed to determine the association between HRT and tear production in postmenopausal women and to observe whether duration of menopause and HRT usage had any effect on the tear quantity.

\section{Methods.}

This cross-sectional study was performed on postmenopausal women, in the department of Obstetrics and Gynecology (OctC), Surgery, Orthopedic and Otorhinolaryngology clinics in UKMMC (Universiti Kebangsaan Malaysia Medical Centre). The subjects comprised of 3 major ethnic races in Malaysia i.e. Malay, Chinese and Indian. These subjects were either with or without HRT. The sample size was calculated from Sample Size Calculations in Clinical Research. A total of 140 patients who were with or without HRT were assessed over a period of 3 months. Prior ethical approval was obtained from the ethics and research committee of the Faculty of Medicine, Universiti Kebangsaan Malaysia (FF-017-2009).

The inclusion criteria were postmenopausal women diagnosed within the last 10 years, with or withoutHRT. Patients with systemic disorders known to cause dry eyes such as Rheumatoid Arthritis, Diabetes Mellitus, Thyroid Disease, AIDS, Graft Versus Host Disease, Parkinson's Disease, patients who had underwent bone marrow transplant, patients on medications known to cause dry eyes like anticholinergic medications, Non-steroidal Anti Inflammatory Drugs, Beta-blocker and radiation therapy, patients with known ocular surface diseases like Herpes Simplex Keratitis and Herpes Zoster Ophthalmicus, topical eye drop users and patients who had recent ocular surgery within 3 months and contact lens wearer were excluded.

Following a written informed consent, a brief ocular and medical history was obtained followed by a brief external eye examination. Schirmer's Test was then performed by trained medical students. Following 5 minutes of topical anesthesia application with Proparacaine Hydrochloride $0.5 \%$, A Schirmer's strip was placed at the inferior conjunctival fornix by placing the bent round wick end of the test strip at the temporal one-third of the lower eyelid margin to avoid corneal touch. The strip was left for 5 minutes while permitting normal blinking or eye closure. The length of the moistened area of the strip was then measured using a millimeter scale. A reading of less than $5 \mathrm{~mm}$ was considered to be dry.

A total of 140 patients with 70 patients in each arm of patients with and without HRT were recruited to make the power of study to be $95 \%$. Data was analyzed using the Statistical Package for Social Science (SPSS) standard version 13.0. T test, chi square and Pearson correlation test was used for data analysis. A value of p<0.05 was considered significant.

\section{Results.}

A total of 140 patients were recruited. There was equal number of participants in both groups of patients with or without HRT. Age ranged from 46 to 74 years old. The mean age was $57.89 \pm 11.89$ years old. The Malays (57.9\%) formed slightly more than half of the study population followed by Chinese $(34.3 \%)$, Indian $(6.4 \%)$ and others (1.4\%, Table 1$)$.

Among the HRT users, almost three quarter (74.3\%) of the patients do not have dry eyes as compared to the non HRT users in which $50 \%$ were found to have dry eyes. This difference was found to be statistically significant $(p=0.003)$ (Table 1, Figure 1).

Among those without HRT, there was no significant association between the occurrence of dry eyes and the duration of menopause. $(r=-0.174, p=0.15)$. For those on HRT treatment, Pearson correlation test also showed no significant association between the tear quantity and duration of HRT consumption ( $r$ value $=-0.133$ and $p=0.271$, Figure 2 and 3 ).

\section{Discussion.}

The role of HRT inhelping tear production is not well understood. Many clinical studies agreed that administration of

Table 1. Demographic Data.

\begin{tabular}{|c|c|c|c|c|}
\hline & Dry eye & Non Dry eye & Total & $p$ value \\
\hline \multicolumn{5}{|l|}{ Age (years) } \\
\hline Mean (SD) & & & $57.89 \pm 11.89$ & \\
\hline Race, n (\%) & & & & 0.870 \\
\hline Malay & & & $81(57.9)$ & \\
\hline Chinese & & & $48(34.3)$ & \\
\hline Indian & & & $9(6.4)$ & \\
\hline Others & & & $2(1.4)$ & \\
\hline HRT, n (\%) & & & & 0.003 \\
\hline HRT Users & $18(25.7)$ & $52(74.3)$ & $70(50)$ & \\
\hline Non HRT users & $35(50.0)$ & $35(50.0)$ & $70(50)$ & \\
\hline
\end{tabular}


estrogen increases both the quantity and quality of tears. ${ }^{8,9}$ Improvement in tear break-up time (TBUT) and Schirmer's test was also reported after the constitution of hormonal therapy. ${ }^{10}$ Lacrimal secretion increases with the usage of combination of estrogen and progesterone treatment. ${ }^{11}$ We includedpatients on all types of HRT whether estrogen alone or combined with progesterone.

Despite many supporting clinical researches on the benefit of HRT on dry eyes symptoms, there was also contradicting data suggesting estrogen as an exacerbating factor for dry eyes. ${ }^{12,13}$ The contradictory results may be attributed to the different tools used to define dry eyes.

Dry eye syndrome is common in the older population. ${ }^{14}$ But it remains difficult to diagnose because of the lack of a gold standard diagnostic testand it is complex etiology and pathophysiology. ${ }^{15}$ Measurement of tear osmolarity might provide a "gold standard" of diagnosis, but a practical tear osmolarity test is not available, to date. ${ }^{16}$

As in the present study, Schirmer's strip is still the most clinically reliable method in managing dry eyes cases. ${ }^{5,6}$ The Schirmer test is widely used clinically for assessing tear production. ${ }^{17,18}$

Figure 1. Comparison of the occurrence of dry eyes between HRT users and non HRT users. There was significant difference between these two groups. HRT users showed significant reduction in the number of patients with dry eyes. $(p=0.003$ )

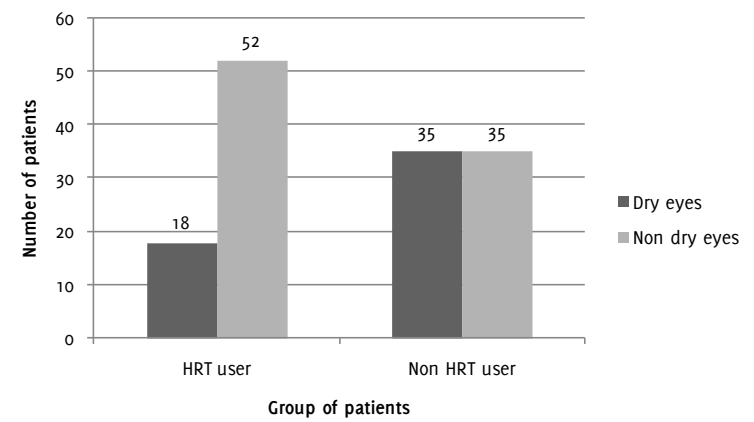

Figure 2. Relationship between duration of menopause in non HRT users with dry eyes. There was no significant association between the occurrence of dry eyes and the duration of menopause. (Pearson correlation test; $r=0.174, p=0.15$ )

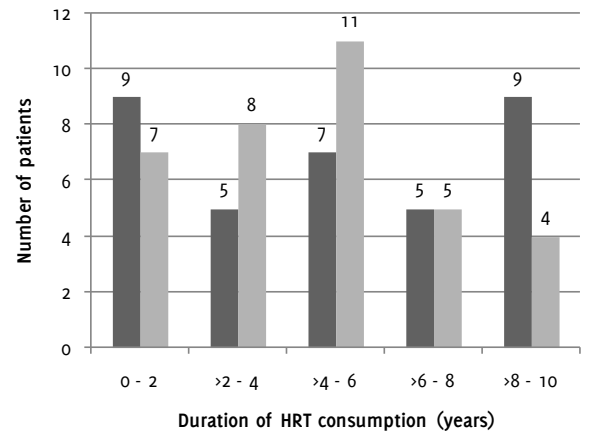

- Dry eyes Non dry eyes
Although there are many small studies which fail toprove the beneficial effect of HRT for dry eyes, the association of menopause and increasing symptoms of dry eyes has been well elaborated. ${ }^{13,19}$ We found higher incidence of dry eyes in menopausal women without HRT treatment as compared to those on hormonal therapy. Even though the duration of treatment was not found to be an important factor in our clinical setting, having HRT on board does help to eliminate dry eyes among post menopausal women in our population.

Argument exists on the fact that the duration of menopause increases dry eyes syndrome..$^{20}$ This does not seem to be true in our population as the duration of menopausedoes not increase the incidence of dry eyes among non HRT user. The findings in the present study support the ocular importance of HRT in elderly women.

Apart from continuous prescription of ophthalmic lubrication, reassurance and discussion on the disease may help to alleviate anxiety and offer additional information for our patients. This cross sectional study however may not be able to elaborate further on the correlation of symptoms and severity of the disease.

Further analytic study in the same setting would be able to evaluate thoroughly the significance of dry eyes in this group of patient. Apart from hormonal factor, consideration of other confounding factors such as surrounding environment and individual social activity would certainly give more useful information as to the effect of prolong HRT consumption or duration of menopause on dry eyes.

In conclusion, HRT use is associated with improved tear production in postmenopausal women. However, the duration of HRT consumption is not associated with improved dry eye condition. Schirmer's test is still a useful clinical method to supplement other diagnostic criteria such as tear break-up time (TBUT) and symptoms-based questionnaire, to further confirm the diagnosis of dry eye.

Figure 2. Relationship between duration of HRT consumption and dry eyes. There was no significant association between the occurrence of dry eyes and duration of HRT consumption. ( $r$ value $=-0.133$ and $p=0.271$ ).

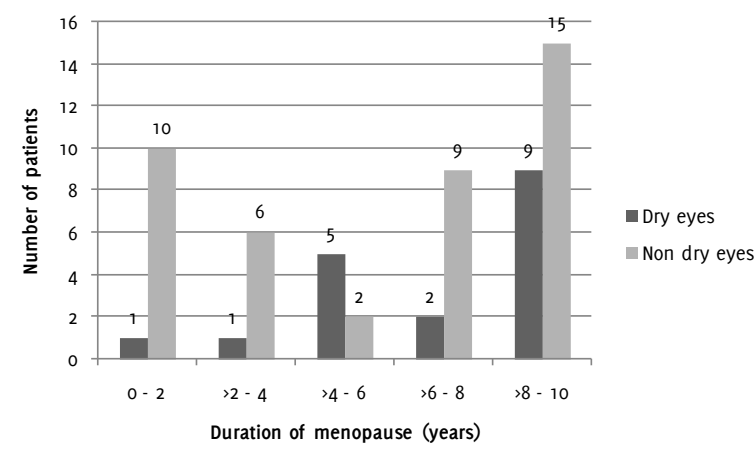




\section{References.}

1. Lemp MA. Report of the National Eye Institute/Industry workshop on Clinical Trials in Dry Eyes. CLAO J 1995;21(4):221-32.

2. Sanchis-Cimeno JA, Lleó-Pérez A, Alonso L, Rahhal MS, Martínez-Soriano F. Reduced corneal thickness values in postmenopausal women with dry eye. Cornea 2005;24(1):39-44.

3. Sullivan DA, Sullivan BD, Evans JE, Schirra F, Yamagami $H$, Liu M, et al. Androgen deficiency, Meibomian gland dysfunction, and evaporative dry eye. Ann N Y Acad Sci 2002;966:211-22.

4. Cenazzani AR, Nicolucci A, Campagnoli C, Crosignani P, Nappi C, Serra CB, et al. Assessment of the QoL in Italian menopausal women: comparison between HRT users and non-users. Maturitas 2002;42(4):267-80.

5. Afonso AA, Monroy D, Stern ME, Feuer W], Tseng SC, Pflugfelder SC. Correlation of tear fluorescein clearance and Schirmer test scores with ocular irritation symptoms. Ophthalmology 1999;106(4):803-10.

6. Xu KP, Tsubota K. Correlation of tear clearance rate and fluorophotometric assessment of tear turnover. Br J Ophthalmol 1995;79(11):1042-5.

7. Danjo Y. Diagnostic usefulness and cutoff value of Schirmer's I test in the Japanese diagnostic criteria of dry eye. Graefes Arch. Clin. Exp. Ophthalmol 1997;235(12):761-6.

8. Athanassiou E, Praidou A, Tzafetas I. Ophthalmic effects of menopause and the role of hormone replacement therapy. Eur Clin Obstet Gynaecol 2007;3(2):63-6.

9. Altintaş O, Caglar Y, Yüksel N, Demirci A, Karabaș L. The effects of menopause and hormone replacement therapy on quality and quantity of tear, intraocular pressure and ocular blood flow. Ophthalmologica 2004;218(2):120-9.

10. Taner P, Akarsu C, Atasoy P, Bayram M, Ergin A. The effects of hormone replacement therapy on ocular surface and tear function tests in postmenopausal women. Ophthalmologica 2004;218(4):257-9.

11. Affinito P, Di Spiezio Sardo A, Di Carlo C, Sammartino A, Tommaselli $\mathrm{GA}$, Bifulco $\mathrm{C}$, et al. Effects of hormone replacement therapy on ocular function in postmenopause. Menopause. 2003;10(5):482-7.

12. Schaumberg DA, Buring JE, Sullivan DA, Dana MR. Hormone replacement therapy and dry eye syndrome. JAMA 2001;286(17):2114-9.

13. Erdem U, Ozdegirmenci O, Sobaci E, Sobaci G, Göktolga U, Dagli S. Dry eye in post-menopausal women using hormone replacement therapy. Maturitas 2007;56(3):257-62.

14. Lekhanont K, Rojanaporn D, Chuck RS, Vongthongsri A. Prevalence of dry eye in Bangkok, Thailand. Cornea 2006;25(10):1162-7.

15. Johnson ME, Murphy PJ. Changes in the tear film and ocular surface from dry eye syndrome. Prog Retin Eye Res 2004;23(4):449-74.

16. Perry HD. Dry eye disease: pathophysiology, classification, and diagnosis. Am J Manag Care 2008;14(3 Suppl):S79-87.

17. Holly FJ. Tear film physiology and contact lens wear. I. Pertinent aspects of tear film physiology. Am J Optom Physiol Opt 1981;58(4):324-30.

18. Shapiro A, Merin S. Schirmer test and break-up time of tear film in normal subjects. Am J Ophthalmol 1979;88(4):752-7.
19. Stadberg E, Mattsson LA, Milsom I. Factors associated with climacteric symptoms and the use of hormone replacement therapy. Acta Obstet Gynecol Scand 2000;79(4):286-92.

20. Marcozzi G, Liberati V, Madia F, Pizzinga A, de Feo G. Effect of hormone replacement therapy on lacrimal fluid peroxidase activity in woman. Maturitas 2003;45(3):225-9.

\footnotetext{
Acknowledgements

We would like to thank Prof. Zainul Rashid bin Mohamad Razi and Ass. Prof. Dr. NorAzlin Ismail from the Department of Obstetric and Gynecology, Ass. Prof. Dr. Razman Jarmin from the Department of Surgery, Prof. Dr. Sharaf Ibrahim from the Department of Orthopaedics, Associate Prof. Dr.Asmabt Abdullah from the Department of Otorhinolaryngology, UKMMC and all the staffs for their cooperation and support.
}

Funding

A grant was received from the Faculty of Medicine fund, Universiti Kebangsaan Malaysia. (FF-017-2009).

Conflict of Interest Statement

The Authors have no conflict of interest to disclose.

Cite as:

Din NM, Sa'aid SHB, Shen LC, Shu YS, Rahman MBA, Aznin ABM, et al. Hormone Replacement Therapy and Dry Eye in Post Menopausal Women: Study in a Tertiary Centre in Malaysia. Int J Med Students 2013;1(1):12-5. 